\title{
Stability of Impulsive Differential Systems
}

\author{
Andrejs Reinfelds ${ }^{1,2}$ and Lelde Sermone ${ }^{2}$ \\ ${ }^{1}$ Institute of Mathematics and Computer Science, University of Latvia, Rainga bulvāris 29, Riga, LV-1459, Latvia \\ ${ }^{2}$ Faculty of Physics and Mathematics, University of Latvia, Zellı iela 8, Riga, LV-1002, Latvia
}

Correspondence should be addressed to Andrejs Reinfelds; reinf@latnet.lv

Received 12 July 2013; Accepted 9 November 2013

Academic Editor: István Györi

Copyright (C) 2013 A. Reinfelds and L. Sermone. This is an open access article distributed under the Creative Commons Attribution License, which permits unrestricted use, distribution, and reproduction in any medium, provided the original work is properly cited.

The asymptotic phase property and reduction principle for stability of a trivial solution is generalized to the case of the noninvertible impulsive differential equations in Banach spaces whose linear parts split into two parts and satisfy the condition of separation.

\section{Introduction}

The reduction principle in the theory of stability for systems of autonomous differential equations for the first time was proved by Pliss [1]. For systems of nonautonomous differential equations it was extended by Aulbach [2]; see also Pötzsche [3]. The analogy of the reduction principle for differential equations in Banach spaces was proved by Lykova [4] and for nonautonomous difference equations in Banach spaces by Reinfelds and Janglajew [5]. Several works $[6,7]$ are devoted to different modifications and applications of the reduction principle. In this paper, we generalize the reduction principle to the case of the noninvertible impulsive differential equations in Banach spaces whose linear part split into two parts and satisfy the condition of separation.

\section{The Statement of the Problem}

Let $\mathbf{X}$ and $\mathbf{Y}$ be Banach spaces. By $\mathfrak{R}(\mathbf{X})$ and $\mathfrak{L}(\mathbf{Y})$ we mean the Banach spaces of bounded linear operators. Consider the following system of impulsive differential equations:

$$
\begin{aligned}
\frac{d x}{d t} & =A(t) x+f(t, x, y), \\
\frac{d y}{d t} & =B(t) y+g(t, x, y), \\
\left.\Delta x\right|_{t=\tau_{i}} & =x\left(\tau_{i}+0\right)-x\left(\tau_{i}-0\right)
\end{aligned}
$$

$$
\begin{aligned}
& =C_{i} x\left(\tau_{i}-0\right)+p_{i}\left(x\left(\tau_{i}-0\right), y\left(\tau_{i}-0\right)\right), \\
\left.\Delta y\right|_{t=\tau_{i}} & =y\left(\tau_{i}+0\right)-y\left(\tau_{i}-0\right) \\
& =D_{i} y\left(\tau_{i}-0\right)+q_{i}\left(x\left(\tau_{i}-0\right), y\left(\tau_{i}-0\right)\right),
\end{aligned}
$$

where

(i) the mappings $A: \mathbb{R} \rightarrow \mathfrak{L}(\mathbf{X})$ and $B: \mathbb{R} \rightarrow \mathfrak{L}(\mathbf{Y})$ are locally integrable in the Bochner sense;

(ii) the mappings $f: \mathbb{R} \times \mathbf{X} \times \mathbf{Y} \rightarrow \mathbf{X}$ and $g: \mathbb{R} \times \mathbf{X} \times$ $\mathbf{Y} \rightarrow \mathbf{Y}$ are locally integrable in the Bochner sense with respect to $t$ for fixed $x$ and $y$, and in addition they satisfy the uniform Lipschitz conditions

$$
\begin{aligned}
& \left|f(t, x, y)-f\left(t, x^{\prime}, y^{\prime}\right)\right| \leq \varepsilon\left(\left|x-x^{\prime}\right|+\left|y-y^{\prime}\right|\right), \\
& \left|g(t, x, y)-g\left(t, x^{\prime}, y^{\prime}\right)\right| \leq \varepsilon\left(\left|x-x^{\prime}\right|+\left|y-y^{\prime}\right|\right) ;
\end{aligned}
$$

(iii) for $i \in \mathbb{Z}, C_{i} \in \mathfrak{L}(\mathbf{X})$, and $D_{i} \in \mathfrak{L}(\mathbf{Y})$, the mappings $p_{i}: \mathbf{X} \times \mathbf{Y} \rightarrow \mathbf{X}$ and $q_{i}: \mathbf{X} \times \mathbf{Y} \rightarrow \mathbf{Y}$ satisfy the uniform Lipschitz conditions

$$
\begin{aligned}
& \left|p_{i}(x, y)-p_{i}\left(x^{\prime}, y^{\prime}\right)\right| \leq \varepsilon\left(\left|x-x^{\prime}\right|+\left|y-y^{\prime}\right|\right), \\
& \left|q_{i}(x, y)-q_{i}\left(x^{\prime}, y^{\prime}\right)\right| \leq \varepsilon\left(\left|x-x^{\prime}\right|+\left|y-y^{\prime}\right|\right) ;
\end{aligned}
$$

(iv) the mappings $x \mapsto x+C_{i} x$ are homeomorphisms; 
(v) the moments $\tau_{i}$ of impulse form a strictly increasing sequence

$$
\cdots<\tau_{-2}<\tau_{-1}<\tau_{0}<\tau_{1}<\tau_{2}<\cdots,
$$

where the limit point may be only $\infty$.

Without loss of generality we assume that the system (1) has the equilibrium points $x=0, y=0$,

$$
\begin{aligned}
& f(t, 0,0)=0, \quad g(t, 0,0)=0, \\
& p_{i}(t, 0,0)=0, \quad q_{i}(t, 0,0)=0 .
\end{aligned}
$$

Using the suitable bump function it is possible for the analysis of local stability of the trivial solution to reduce to investigation of the global stability of the trivial solution if the nonlinear terms of (1) are uniform Lipschitz with respect to time and with a sufficient small constant in a fixed radius tubular neighbourhood of the trivial solution.

For simplicity, we assume that the linear part of (1) is decoupled in two separate parts. In many cases, this can be reached via the so-called kinematic similarity transformation [8, 9]. More generally via kinematic similarity transformation, the linear system can be reduced to the same almost reducible system [10], a system with a diagonal part and a small nondiagonal part. However, the kinematic transformation can grow unboundedly as the nondiagonal part tends to zero.

Definition 1 (see $[11,12])$. By the solution to an impulsive system one means a piecewise absolutely continuous mapping with discontinuities of the first kind at the points $t=\tau_{i}$ which for almost all $t$ satisfies system (1) and for $t=\tau_{i}$ satisfies the conditions of a "jump."

Note that condition (v) together with the Lipschitz property with respect to $x$ and $y$ of the right-hand side ensures that there is a unique solution.

Let $\Phi(\cdot, s, x, y)=(x(\cdot, s, x, y), y(\cdot, s, x, y)):[s,+\infty) \rightarrow$ $\mathbf{X} \times \mathbf{Y}$ be the solution of system (1), where $\Phi(s+0, s, x, y)=$ $(x(s+0, s, x, y), y(s+0, s, x, y))=(x, y)$. At the break points $\tau_{i}$ the values for all solutions are taken at $\tau_{i}+0$ unless otherwise indicated. For short, we will use the notation $\Phi(t)=(x(t), y(t))$.

Let $X(t, s)$ and $Y(t, s)$ be the evolutionary operators of the impulsive linear differential equations

$$
\begin{aligned}
& \frac{d x}{d t}=A(t) x, \\
& \left.\Delta x\right|_{t=\tau_{i}}=x\left(\tau_{i}+0\right)-x\left(\tau_{i}-0\right)=C_{i} x\left(\tau_{i}-0\right),
\end{aligned}
$$

and, respectively,

$$
\begin{aligned}
& \frac{d y}{d t}=B(t) y, \\
& \left.\Delta y\right|_{t=\tau_{i}}=y\left(\tau_{i}+0\right)-y\left(\tau_{i}-0\right)=D_{i} y\left(\tau_{i}-0\right) .
\end{aligned}
$$

We assume that the operators $X(t, s)$ and $Y(t, s)$ satisfy the condition of separation [7]:

$$
\begin{aligned}
v=\max \left(\sup _{s} \int_{-\infty}^{s}|Y(s, t)||X(t, s)| d t\right. \\
\quad+\sup _{s} \sum_{\tau_{i} \leq s}\left|Y\left(s, \tau_{i}\right)\right|\left|X\left(\tau_{i}-0, s\right)\right|, \\
\quad \sup _{s} \int_{s}^{+\infty}|X(s, t)||Y(t, s)| d t \\
\left.\quad+\sup _{s} \sum_{s<\tau_{i}}\left|X\left(s, \tau_{i}\right)\right|\left|Y\left(\tau_{i}-0, s\right)\right|\right)<+\infty,
\end{aligned}
$$

where $v$ is the constant of separation.

To prove the theorems and lemmas, we use integrals which include evolutionary operators in their integrands. That is why it is more useful to estimate not the evolutionary operators but the corresponding integrals. Doing so, on the one hand, the conditions of theorems and lemmas are released from unnecessary technical limitations and, on the other hand, we obtain the conditions that are close to the necessary conditions.

If $A(t)=A, B(t)=B, C_{i}=0$, and $D_{i}=0$, then $v=\int_{0}^{+\infty}\left|e^{-A t}\right|\left|e^{B t}\right| d t$. Consequently, the integral converges if the spectrum of the mapping $B$ is located to the left of the spectrum of the mapping $A$ and the spectra are separated by a vertical line in the complex plane.

Let $\mathbf{P C}(\mathbb{R} \times \mathbf{X}, \mathbf{Y})$ be a set of mappings $u: \mathbb{R} \times \mathbf{X} \rightarrow$ $\mathbf{Y}$ that are continuous for $(t, x) \in\left[\tau_{i}, \tau_{i+1}\right) \times \mathbf{X}$ and have discontinuities of the first kind for $t=\tau_{i}$.

The set

$$
\begin{gathered}
\mathfrak{M}=\{u \in \mathbf{P C}(\mathbb{R} \times \mathbf{X}, \mathbf{Y}) \mid u(s, 0)=0, \\
\left.\sup _{s, x \neq 0} \frac{|u(s, x)|}{|x|}<+\infty\right\}
\end{gathered}
$$

is a Banach space with the norm

$$
\begin{gathered}
\|u\|=\sup _{s, x \neq 0} \frac{|u(s, x)|}{|x|}, \\
\mathfrak{M}(k)=\{u \in \mathfrak{M} \mid\|u\| \leq k, \\
\left.\left|u(s, x)-u\left(s, x^{\prime}\right)\right| \leq k\left|x-x^{\prime}\right|\right\}
\end{gathered}
$$

are a closed subsets of $\mathbf{M}$. 


\section{Auxiliary Lemma}

Lemma 2. Let $u, u^{\prime} \in \mathfrak{M}(k)$ and $\varepsilon v(k+1)<1$. Then the following estimations are valid:

$$
\begin{aligned}
\int_{-\infty}^{s} & |Y(s, t)||z(t)| d t+\sum_{\tau_{i} \leq s}\left|Y\left(s, \tau_{i}\right)\right|\left|z\left(\tau_{i}-0\right)\right| \\
\leq & \frac{v|x|}{1-\varepsilon v(k+1)}, \\
\int_{-\infty}^{s} & |Y(s, t)|\left|z(t)-z^{\prime}(t)\right| d t \\
& +\sum_{\tau_{i} \leq s}\left|Y\left(s, \tau_{i}\right)\right|\left|z\left(\tau_{i}-0\right)-z^{\prime}\left(\tau_{i}-0\right)\right| \\
\leq & \frac{v}{1-\varepsilon v(k+1)} \\
& \times\left(\left|x-x^{\prime}\right|+\frac{\varepsilon v}{1-\varepsilon v(k+1)}|x|\left\|u-u^{\prime}\right\|\right),
\end{aligned}
$$

where $z: \mathbb{R} \rightarrow \mathbf{X}$ is the solution of the impulsive differential equations

$$
\begin{aligned}
& \frac{d z}{d t}=A(t) z+f(t, z, u(t, z)) \\
& \left.\Delta z\right|_{t=\tau_{i}}=C_{i} z\left(\tau_{i}-0\right)+p_{i}\left(z\left(\tau_{i}-0\right), u\left(\tau_{i}-0, z\left(\tau_{i}-0\right)\right)\right)
\end{aligned}
$$

satisfying the initial condition $z(s)=x$.

We remark that $X\left(\tau_{i}-0, \tau_{i}\right)=\left(i d_{x}+C_{i}\right)^{-1}$ and $\mid X\left(\tau_{i}-\right.$ $\left.0, \tau_{i}\right) \mid \leq \nu$. It follows that (12) has a unique backward solution if $\varepsilon(k+1) v<1$.

Proof. The solution of the impulsive system (12) for $t \leq s$ is

$$
\begin{aligned}
z(t)= & X(t, s) x+\int_{s}^{t} X(t, \tau) f(\tau, z(\tau), u(\tau, z(\tau))) d \tau \\
& -\sum_{t<\tau_{i} \leq s} X\left(t, \tau_{i}\right) p_{i}\left(z\left(\tau_{i}-0\right), u\left(\tau_{i}-0, z\left(\tau_{i}-0\right)\right)\right) .
\end{aligned}
$$

Taking into account that $f$ and $p_{i}$ satisfy the uniform Lipschitz conditions and $u$ properties, the solution $z(t)$ can be estimated by

$$
\begin{aligned}
|z(t)| \leq & |X(t, s)||x| \\
& +\varepsilon(k+1)\left(\int_{t}^{s}|X(t, \tau)||z(\tau)| d \tau\right. \\
& \left.\quad+\sum_{t<\tau_{i} \leq s}\left|X\left(t, \tau_{i}\right)\right|\left|z\left(\tau_{i}-0\right)\right|\right) .
\end{aligned}
$$

Multiplying the solution $z(t)$ by $|Y(s, t)|$ and integrating from $-\infty$ to $s$, we obtain

$$
\begin{aligned}
& \int_{-\infty}^{s}|Y(s, t)||z(t)| d t \leq|x| \int_{-\infty}^{s}|Y(s, t)||X(t, s)| d t \\
& \quad+\varepsilon(k+1) \sup _{\tau} \int_{-\infty}^{\tau}|Y(\tau, t)||X(t, \tau)| d t \\
& \quad \times\left(\int_{-\infty}^{s}|Y(s, \tau)||z(\tau)| d \tau+\sum_{\tau_{i} \leq s}\left|Y\left(s, \tau_{i}\right)\right|\left|z\left(\tau_{i}-0\right)\right|\right) .
\end{aligned}
$$

Multiplying $z\left(\tau_{i}-0\right)$ by $\left|Y\left(s, \tau_{i}\right)\right|$ and summing for all $i$ with respect to $\tau_{i} \leq s$, we obtain

$$
\begin{aligned}
& \sum_{\tau_{i} \leq s}\left|Y\left(s, \tau_{i}\right)\right|\left|z\left(\tau_{i}-0\right)\right| \\
& \leq|x| \sum_{\tau_{i} \leq s}\left|Y\left(s, \tau_{i}\right)\right|\left|X\left(\tau_{i}-0, s\right)\right| \\
& \quad+\varepsilon(k+1) \sup _{\tau} \sum_{\tau_{i} \leq \tau}\left|Y\left(\tau, \tau_{i}\right)\right|\left|X\left(\tau_{i}-0, \tau\right)\right| \\
& \quad \times\left(\int_{-\infty}^{s}|Y(s, \tau)||z(\tau)| d \tau+\sum_{\tau_{i} \leq s}\left|Y\left(s, \tau_{i}\right)\right|\left|z\left(\tau_{i}-0\right)\right|\right) .
\end{aligned}
$$

Summing up we get that

$$
\begin{aligned}
\int_{-\infty}^{s} & |Y(s, \tau)||z(\tau)| d \tau+\sum_{\tau_{i} \leq s}\left|Y\left(s, \tau_{i}\right)\right|\left|z\left(\tau_{i}-0\right)\right| \\
\leq & \nu|x|+\varepsilon \nu(k+1) \\
\quad & \times\left(\int_{-\infty}^{s}|Y(s, \tau)||z(\tau)| d \tau+\sum_{\tau_{i} \leq s}\left|Y\left(s, \tau_{i}\right)\right|\left|z\left(\tau_{i}-0\right)\right|\right) .
\end{aligned}
$$

From the last inequality, we get that

$$
\begin{aligned}
& \int_{-\infty}^{s}|Y(s, \tau)||z(\tau)| d \tau+\sum_{\tau_{i} \leq s}\left|Y\left(s, \tau_{i}\right)\right|\left|z\left(\tau_{i}-0\right)\right| \\
& \quad \leq \frac{\nu|x|}{1-\nu \varepsilon(k+1)} .
\end{aligned}
$$


Now we estimate the difference $\left|z(t)-z^{\prime}(t)\right|$ taking into consideration the properties of $f, p_{i}$, and $u$ :

$$
\begin{aligned}
& \left|z(t)-z^{\prime}(t)\right| \\
& \leq|X(t, s)|\left|x-x^{\prime}\right|+\varepsilon(k+1) \\
& \quad \times\left(\int_{t}^{s}|X(t, \tau)|\left|z(\tau)-z^{\prime}(\tau)\right| d \tau\right. \\
& \left.\quad+\sum_{t<\tau_{i} \leq s}\left|X\left(t, \tau_{i}\right)\right|\left|z\left(\tau_{i}-0\right)-z^{\prime}\left(\tau_{i}-0\right)\right|\right) \\
& +\varepsilon\left\|u-u^{\prime}\right\|\left(\int_{t}^{s}|X(t, \tau)||z(\tau)| d \tau\right. \\
& \left.\quad+\sum_{t<\tau_{i} \leq s}\left|X\left(t, \tau_{i}\right)\right|\left|z\left(\tau_{i}-0\right)\right|\right) .
\end{aligned}
$$

Multiplying the difference $\left|z(t)-z^{\prime}(t)\right|$ by $|Y(s, t)|$ and integrating from $-\infty$ to $s$, we obtain

$$
\begin{aligned}
& \int_{-\infty}^{s}|Y(s, t)|\left|z(t)-z^{\prime}(t)\right| d t \\
& \leq\left|x-x^{\prime}\right| \int_{-\infty}^{s}|Y(s, t)||X(t, s)| d t \\
& \quad+\varepsilon(k+1) \sup _{\tau} \int_{-\infty}^{\tau}|Y(\tau, t)||X(t, \tau)| d t \\
& \quad \times\left(\int_{-\infty}^{s}|Y(s, \tau)|\left|z(\tau)-z^{\prime}(\tau)\right| d \tau\right. \\
& \left.\quad+\sum_{\tau_{i} \leq s}\left|Y\left(s, \tau_{i}\right)\right|\left|z\left(\tau_{i}-0\right)-z^{\prime}\left(\tau_{i}-0\right)\right|\right) \\
& +\varepsilon\left\|u-u^{\prime}\right\| \sup _{\tau} \int_{-\infty}^{\tau}|Y(\tau, t)||X(t, \tau)| d t \\
& \quad \times\left(\int_{-\infty}^{s}|Y(s, \tau)||z(\tau)| d \tau+\sum_{\tau_{i} \leq s}\left|Y\left(s, \tau_{i}\right)\right|\left|z\left(\tau_{i}-0\right)\right|\right) .
\end{aligned}
$$

Multiplying the difference $\left|z\left(\tau_{i}-0\right)-z^{\prime}\left(\tau_{i}-0\right)\right|$ by $\left|Y\left(s, \tau_{i}\right)\right|$ and summing for all $i$ with respect to $\tau_{i} \leq s$, we obtain

$$
\begin{aligned}
& \sum_{\tau_{i} \leq s}\left|Y\left(s, \tau_{i}\right)\right|\left|z\left(\tau_{i}-0\right)-z^{\prime}\left(\tau_{i}-0\right)\right| \\
& \leq\left|x-x^{\prime}\right| \sum_{\tau_{i} \leq s}\left|Y\left(s, \tau_{i}\right)\right|\left|X\left(\tau_{i}-0, s\right)\right| \\
& \quad+\varepsilon(k+1) \sup _{\tau} \sum_{\tau_{i} \leq \tau}\left|Y\left(\tau, \tau_{i}\right)\right|\left|X\left(\tau_{i}-0, \tau\right)\right|
\end{aligned}
$$

$$
\begin{aligned}
& \times\left(\int_{-\infty}^{s}|Y(s, \tau)|\left|z(\tau)-z^{\prime}(\tau)\right| d \tau\right. \\
& \left.\quad+\sum_{\tau_{i} \leq s}\left|Y\left(s, \tau_{i}\right)\right|\left|z\left(\tau_{i}-0\right)-z^{\prime}\left(\tau_{i}-0\right)\right|\right) \\
& +\varepsilon\left\|u-u^{\prime}\right\| \sup _{\tau} \sum_{\tau_{i} \leq \tau}\left|Y\left(\tau, \tau_{i}\right)\right|\left|X\left(\tau_{i}-0, \tau\right)\right| \\
& \times\left(\int_{-\infty}^{s}|Y(s, \tau)||z(\tau)| d \tau\right. \\
& \left.\quad+\sum_{\tau_{i} \leq s}\left|Y\left(s, \tau_{i}\right)\right|\left|z\left(\tau_{i}-0\right)\right|\right) .
\end{aligned}
$$

Summing up, we get that

$$
\begin{aligned}
& \int_{-\infty}^{s}|Y(s, \tau)|\left|z(\tau)-z^{\prime}(\tau)\right| d \tau \\
& +\sum_{\tau_{i} \leq s}\left|Y\left(s, \tau_{i}\right)\right|\left|z\left(\tau_{i}-0\right)-z^{\prime}\left(\tau_{i}-0\right)\right| \\
& \leq v\left(\left|x-x^{\prime}\right|+\varepsilon(k+1)\right. \\
& \quad \times\left(\int_{-\infty}^{s}|Y(s, \tau)|\left|z(\tau)-z^{\prime}(\tau)\right| d \tau\right. \\
& \left.\left.\quad+\sum_{\tau_{i} \leq s}\left|Y\left(s, \tau_{i}\right)\right|\left|z\left(\tau_{i}-0\right)-z^{\prime}\left(\tau_{i}-0\right)\right|\right)\right) \\
& +\varepsilon v\left\|u-u^{\prime}\right\| \\
& \times\left(\int_{-\infty}^{s}|Y(s, \tau)||z(\tau)| d \tau\right. \\
& \left.\quad+\sum_{\tau_{i} \leq s}\left|Y\left(s, \tau_{i}\right)\right|\left|z\left(\tau_{i}-0\right)\right|\right) .
\end{aligned}
$$

Applying the first result of Lemma 2, we get

$$
\begin{aligned}
& \int_{-\infty}^{s}|Y(s, \tau)|\left|z(\tau)-z^{\prime}(\tau)\right| d \tau \\
& +\sum_{\tau_{i} \leq s}\left|Y\left(s, \tau_{i}\right)\right|\left|z\left(\tau_{i}-0\right)-z^{\prime}\left(\tau_{i}-0\right)\right| \\
& \leq v\left(\left|x-x^{\prime}\right|+\varepsilon(k+1)\right. \\
& \quad \times\left(\int_{-\infty}^{s}|Y(s, \tau)|\left|z(\tau)-z^{\prime}(\tau)\right| d \tau\right. \\
& \left.\left.\quad+\sum_{\tau_{i} \leq s}\left|Y\left(s, \tau_{i}\right)\right|\left|z\left(\tau_{i}-0\right)-z^{\prime}\left(\tau_{i}-0\right)\right|\right)\right) \\
& +\varepsilon v\left\|u-u^{\prime}\right\| \frac{\nu|x|}{1-\varepsilon v(k+1)} .
\end{aligned}
$$

From the last inequality we easily obtain (11). 


\section{Existence of a Lipschitz Invariant Manifold}

Theorem 3. If $4 \varepsilon v<1$, then there exists a unique piecewise continuous mapping $u \in \mathfrak{M}(k)$ satisfying the following properties:

(i) $u(t, x(t, s, x, u(s, x)))=y(t, s, x, u(s, x))$ for $t \geq s$;

(ii) $\left|u(s, x)-u\left(s, x^{\prime}\right)\right| \leq k\left|x-x^{\prime}\right|$;

(iii) $u(t, 0)=0$.

Proof. Consider in $\mathfrak{M}(k)$ the functional equation

$$
\begin{aligned}
& u(s, x) \\
& =\int_{-\infty}^{s} Y(s, \tau) g(\tau, z(\tau), u(\tau, z(\tau))) d \tau \\
& \quad+\sum_{\tau_{i} \leq s} Y\left(s, \tau_{i}\right) q_{i}\left(z\left(\tau_{i}-0\right), u\left(\tau_{i}-0, z\left(\tau_{i}-0\right)\right)\right),
\end{aligned}
$$

where $z: \mathbb{R} \rightarrow \mathbf{X}$ is the solution of the impulsive differential equation system (12) satisfying the initial condition $z(s)=x$.

Consider the operator $\mathscr{L}: \mathfrak{M}(k) \rightarrow \mathfrak{M}(k)$ defined by the formula

$$
\begin{aligned}
\mathscr{L} u(s, x) \\
=\int_{-\infty}^{s} Y(s, \tau) g(\tau, z(\tau), u(\tau, z(\tau))) d \tau \\
\quad+\sum_{\tau_{i} \leq s} Y\left(s, \tau_{i}\right) q_{i}\left(z\left(\tau_{i}-0\right), u\left(\tau_{i}-0, z\left(\tau_{i}-0\right)\right)\right) .
\end{aligned}
$$

If $4 \varepsilon v<1$, then

$$
k=(2 \varepsilon v)^{-1}(1-2 \varepsilon \nu-\sqrt{1-4 \varepsilon v})=\frac{1-\sqrt{1-4 \varepsilon v}}{1+\sqrt{1-4 \varepsilon v}}<1
$$

satisfies the equality

$$
k=\varepsilon \mathcal{v}(k+1)(1-\varepsilon \mathcal{v}(k+1))^{-1} .
$$

Then

$$
\begin{aligned}
|\mathscr{L} u(s, x)| \\
\leq \varepsilon(k+1) \\
\quad\left(\int_{-\infty}^{s}|Y(s, \tau)||z(\tau)| d \tau+\sum_{\tau_{i} \leq s}\left|Y\left(s, \tau_{i}\right)\right|\left|z\left(\tau_{i}-0\right)\right|\right) \\
\leq \frac{\varepsilon v(k+1)|x|}{1-\varepsilon \nu(k+1)}=k|x| .
\end{aligned}
$$

It follows that $\|\mathscr{L} u\| \leq k$.
Taking into account that $g$ and $q_{i}$ satisfy the uniform Lipschitz conditions, we get that

$$
\begin{aligned}
& \left|\mathscr{L} u(s, x)-\mathscr{L} u^{\prime}\left(s, x^{\prime}\right)\right| \\
& \leq \varepsilon(k+1) \\
& \quad \times\left(\int_{-\infty}^{s}|Y(s, \tau)|\left|z(\tau)-z^{\prime}(\tau)\right| d \tau\right. \\
& \left.\quad+\sum_{\tau_{i} \leq s}\left|Y\left(s, \tau_{i}\right)\right|\left|z\left(\tau_{i}-0\right)-z^{\prime}\left(\tau_{i}-0\right)\right|\right) \\
& \quad+\varepsilon\left\|u-u^{\prime}\right\|\left(\int_{-\infty}^{s}|Y(s, \tau)||z(\tau)| d \tau\right. \\
& \quad+\frac{\left.+\sum_{\tau_{i} \leq s}\left|Y\left(s, \tau_{i}\right)\right|\left|z\left(\tau_{i}-0\right)\right|\right)}{1-\varepsilon v(k+1)}\left\|u-u^{\prime}\right\| \\
& =k\left|x-x^{\prime}\right|+k|x|\left\|u-u^{\prime}\right\| .
\end{aligned}
$$

We have that $\mathscr{L} u \in \mathfrak{M}(k)$ and $\mathscr{L}$ is a contraction in $\mathfrak{M}(k)$, and therefore there is only one solution satisfying the functional equation $\mathscr{L} u=u$.

In addition for $t \geq s$

$$
\begin{aligned}
& u(t, z(t)) \\
& =\int_{-\infty}^{t} Y(t, \tau) g(\tau, z(\tau), u(\tau, z(\tau))) d \tau \\
& \quad+\sum_{\tau_{i} \leq t} Y\left(t, \tau_{i}\right) q_{i}\left(z\left(\tau_{i}-0\right), u\left(\tau_{i}-0, z\left(\tau_{i}-0\right)\right)\right) \\
& =Y(t, s) u(s, x)+\int_{s}^{t} Y(t, \tau) g(\tau, z(\tau), u(\tau, z(\tau))) d \tau \\
& \quad+\sum_{s<\tau_{i} \leq t} Y\left(t, \tau_{i}\right) q_{i}\left(z\left(\tau_{i}-0\right), u\left(\tau_{i}-0, z\left(\tau_{i}-0\right)\right)\right) .
\end{aligned}
$$

Therefore for uniqueness of solutions we get for $t \geq s$

$$
\begin{aligned}
& z(t)=x(t, s, x, u(s, x)), \\
& u(t, z(t))=y(t, s, x, u(s, x)) .
\end{aligned}
$$




\section{Behaviour of Solutions in the Neighbourhood of an Invariant Manifold}

Theorem 4. Let $4 \varepsilon v<1$. Then the following estimation is valid:

$$
\begin{aligned}
\int_{s}^{+\infty} & |X(s, t)||y(t)-u(t, x(t))| d t \\
& +\sum_{s<\tau_{i}}\left|X\left(s, \tau_{i}\right)\right|\left|y\left(\tau_{i}-0\right)-u\left(\tau_{i}-0, x\left(\tau_{i}-0\right)\right)\right| \\
& \leq \frac{\nu|y-u(s, x)|}{1-\varepsilon v(k+1)}
\end{aligned}
$$

The inequality characterizes the integral distance between an arbitrary solution and an invariant manifold.

Proof. For an arbitrary map $\xi: \mathbb{R} \rightarrow \mathbf{Y}$, piecewise continuous from the right with points of discontinuity $t=\tau_{i}$ of the first type, we have the following relation:

$$
\xi(t)=\xi(s)+\lim _{\delta \rightarrow+0} \frac{1}{\delta} \int_{s}^{t}(\xi(r+\delta)-\xi(r)) d r .
$$

Set $\xi(r)=Y(t, r) u(r, x(r))$. Then for $t \geq s$ we obtain

$$
\begin{aligned}
u(t, x(t))= & Y(t, s) u(s, x) \\
& +\lim _{\delta \rightarrow+0} \frac{1}{\delta} \int_{s}^{t}(Y(t, r+\delta) u(r+\delta, x(r+\delta)) \\
= & Y(t, s) u(s, x) \\
& \left.+\lim _{\delta \rightarrow+0} \frac{1}{\delta} \int_{s}^{t} Y(t, r+\delta) u(r, x(r))\right) d r \\
& \quad \times(u(r+\delta, x(r+\delta)) \\
& +\lim _{\delta \rightarrow+0} \frac{1}{\delta} \int_{s}^{t}(Y(t, r+\delta) y \\
& \quad \times(r+\delta, r, x(r), u(r, x(r))) \\
& \quad-Y(t, r) u(r, x(r))) d r .
\end{aligned}
$$

Let us note that

$$
\begin{aligned}
y(r+ & \left.\delta, r, x_{1}, y_{1}\right) \\
= & Y(r+\delta, r) y_{1} \\
& +\int_{r}^{r+\delta} Y(r+\delta, \tau) g\left(\tau, \Phi\left(\tau, r, x_{1}, y_{1}\right)\right) d \tau \\
& +\sum_{r<\tau_{i} \leq r+\delta} Y\left(r+\delta, \tau_{i}\right) q_{i}\left(\Phi\left(\tau_{i}-0, r, x_{1}, y_{1}\right)\right) .
\end{aligned}
$$

The third countable can be simplified:

$$
\begin{gathered}
\lim _{\delta \rightarrow+0} \frac{1}{\delta} \int_{s}^{t}\left(\int_{r}^{r+\delta} Y(t, \tau) g(\tau, \Phi(\tau, r, x(r), u(r, x(r)))) d \tau\right. \\
\quad+\sum_{r<\tau_{i} \leq r+\delta} Y\left(t, \tau_{i}\right) q_{i} \\
\left.\quad \times\left(\Phi\left(\tau_{i}-0, r, x(r), u(r, x(r))\right)\right)\right) d r \\
=\int_{s}^{t} Y(t, r) g(r, x(r), u(r, x(r))) d r \\
+\sum_{s<\tau_{i} \leq t} Y\left(t, \tau_{i}\right) q_{i}\left(x\left(\tau_{i}-0\right), u\left(\tau_{i}-0, x\left(\tau_{i}-0\right)\right)\right) .
\end{gathered}
$$

Next we obtain

$$
\begin{aligned}
& y(t)-u(t, x(t)) \\
&= Y(t, s)(y-u(s, x)) \\
&+\int_{s}^{t} Y(t, r)(g(r, x(r), y(r)) \\
&-g(r, x(r), u(r, x(r)))) d r \\
&+ \lim _{\delta \rightarrow+0} \frac{1}{\delta} \int_{s}^{t} Y(t, r+\delta) \\
& \times(y(r+\delta, r, x(r), u(r, x(r))) \\
&+-u(r+\delta, x(r+\delta))) d r \\
& \sum_{s<\tau_{i} \leq t} Y\left(t, \tau_{i}\right) \\
& \times\left(q_{i}\left(x\left(\tau_{i}-0\right), y\left(\tau_{i}-0\right)\right)\right. \\
&\left.-q_{i}\left(x\left(\tau_{i}-0\right), u\left(\tau_{i}-0, x\left(\tau_{i}-0\right)\right)\right)\right) .
\end{aligned}
$$

Now we consider

$$
x\left(r+\delta, r, x_{1}, y_{1}\right)-x\left(r+\delta, r, x_{1}, u\left(r, x_{1}\right)\right)
$$

$$
\begin{gathered}
=\int_{r}^{r+\delta} X(r+\delta, \tau)\left(f\left(\tau, \Phi\left(\tau, r, x_{1}, y_{1}\right)\right)\right. \\
\left.-f\left(\tau, \Phi\left(\tau, r, x_{1}, u\left(r, x_{1}\right)\right)\right)\right) d \tau \\
+\sum_{r<\tau_{i} \leq r+\delta} X\left(r+\delta, \tau_{i}\right)\left(p_{i}\left(\Phi\left(\tau_{i}-0, r, x_{1}, y_{1}\right)\right)\right. \\
\left.-p_{i}\left(\Phi\left(\tau_{i}-0, r, x_{1}, u\left(r, x_{1}\right)\right)\right)\right) .
\end{gathered}
$$


Thus,

$$
\begin{aligned}
& \lim _{\delta \rightarrow+0} \frac{1}{\delta} \mid \int_{s}^{t} Y(t, r+\delta)(y(r+\delta, r, x(r), u(r, x(r))) \\
& -u(r+\delta, x(r+\delta))) d r \\
& \leq k \lim _{\delta \rightarrow+0} \frac{1}{\delta} \\
& \times \int_{s}^{t}|Y(t, r+\delta)| \\
& \times \mid x(r+\delta, r, x(r), u(r, x(r))) \\
& -x(r+\delta, r, x(r), y(r)) \mid d r \\
& \leq k \lim _{\delta \rightarrow+0} \frac{1}{\delta} \\
& \times \int_{s}^{t}|Y(t, r+\delta)| \\
& \times\left(\int_{r}^{r+\delta}|X(r+\delta, \tau)|\right. \\
& \times \mid f(\tau, \Phi(\tau, r, x(r), y(r))) \\
& -f(\tau, \Phi(\tau, r, x(r) u(r, x(r)))) \mid d \tau \\
& +\sum_{r<\tau_{i} \leq r+\delta}\left|X\left(r+\delta, \tau_{i}\right)\right| \\
& \times \mid p_{i}\left(\Phi\left(\tau_{i}-0, r, x(r), y(r)\right)\right) \\
& \left.-p_{i}\left(\Phi\left(\tau_{i}-0, r, x(r), u(r, x(r))\right)\right) \mid\right) d r \\
& =k\left(\int_{s}^{t}|Y(t, r)|\right. \\
& \times|f(r, x(r), y(r))-f(r, x(r), u(r, x(r)))| d r \\
& +\sum_{s<\tau_{i} \leq t}\left|Y\left(t, \tau_{i}\right)\right| \\
& \times \mid p_{i}\left(x\left(\tau_{i}-0\right), y\left(\tau_{i}-0\right)\right) \\
& \left.-p_{i}\left(x\left(\tau_{i}-0\right), u\left(\tau_{i}-0, x\left(\tau_{i}-0\right)\right)\right) \mid\right) .
\end{aligned}
$$

We introduce the expression $\eta(t)=|y(t)-u(t, x(t))|$. For $t \geq s$, we obtain the estimation

$$
\begin{aligned}
\eta(t) \leq & |Y(t, s)| \eta(s) \\
& +\varepsilon(k+1) \\
& \times\left(\int_{s}^{t}|Y(t, r)| \eta(r) d r+\sum_{s<\tau_{i} \leq t}\left|Y\left(t, \tau_{i}\right)\right| \eta\left(\tau_{i}-0\right)\right) .
\end{aligned}
$$

Multiplying by $X(s, t)$, integrating, and summing analogously as in auxiliary Lemma 2, we obtain the inequality

$$
\begin{aligned}
\int_{s}^{+\infty} & |X(s, t)||y(t)-u(t, x(t))| d t \\
& +\sum_{s<\tau_{i}}\left|X\left(s, \tau_{i}\right)\right|\left|y\left(\tau_{i}-0\right)-u\left(\tau_{i}-0, x\left(\tau_{i}-0\right)\right)\right| \\
& \leq \frac{\nu|y-u(s, x)|}{1-\varepsilon \nu(k+1)} .
\end{aligned}
$$

\section{Asymptotic Phase Type Property}

Theorem 5. Let $4 \varepsilon v<1$. Then for every solution $(x(\cdot), y(\cdot))$ : $[s,+\infty) \rightarrow \mathbf{X} \times \mathbf{Y}$ of the impulsive system (1) there is a such solution $\zeta(\cdot):[s,+\infty) \rightarrow \mathbf{X}$ of the impulsive system (12) that for all $t \geq s$ the following estimation is fulfilled:

$$
|\zeta(t)-x(t)| \leq k_{1}|y(t)-u(t, x(t))|
$$

where

$$
k_{1}=\frac{\varepsilon \nu}{\sqrt{1-4 \varepsilon v}}
$$

Proof. The set of mappings

$$
\mathfrak{M}_{1}=\left\{\kappa \in \mathbf{P C}(\mathbb{R} \times \mathbf{X} \times \mathbf{Y}, \mathbf{X}) \mid \sup _{s, x, y} \frac{|\kappa(s, x, y)|}{|y-u(s, x)|}<+\infty\right\}
$$

is a Banach space with the norm

$$
\|\kappa\|=\sup _{s, x, y} \frac{|\kappa(s, x, y)|}{|y-u(s, x)|}
$$

respectively.

Consider the functional equation in $\mathfrak{M}_{1}$

$$
\begin{aligned}
& \kappa(s, x, y) \\
& =\int_{s}^{+\infty} X(s, \tau)(f(\tau, \Phi(\tau)) \\
& -f(\tau, x(\tau)+\kappa(\tau, \Phi(\tau)), \\
& u(\tau, x(\tau)+\kappa(\tau, \Phi(\tau))))) d \tau \\
& +\sum_{s<\tau_{i}} X\left(s, \tau_{i}\right) \\
& \times\left(p_{i}\left(\Phi\left(\tau_{i}-0\right)\right)\right. \\
& -p_{i}\left(x\left(\tau_{i}-0\right)+\kappa\left(\tau_{i}-0, \Phi\left(\tau_{i}-0\right)\right),\right. \\
& u\left(\tau_{i}-0, x\left(\tau_{i}-0\right)\right. \\
& \left.\left.\left.+\kappa\left(\tau_{i}-0, \Phi\left(\tau_{i}-0\right)\right)\right)\right)\right) .
\end{aligned}
$$


Consider the operator $\mathscr{L}: \mathfrak{M}_{1} \rightarrow \mathfrak{M}_{1}$ defined by formula

$$
\begin{aligned}
& \mathscr{L} \kappa(s, x, y) \\
& =\int_{s}^{+\infty} X(s, \tau)(f(\tau, \Phi(\tau)) \\
& -f(\tau, x(\tau)+\kappa(\tau, \Phi(\tau)) \\
& u(\tau, x(\tau)+\kappa(\tau, \Phi(\tau))))) d \tau \\
& +\sum_{s<\tau_{i}} X\left(s, \tau_{i}\right) \\
& \times\left(p_{i}\left(\Phi\left(\tau_{i}-0\right)\right)\right. \\
& -p_{i}\left(x\left(\tau_{i}-0\right)+\kappa\left(\tau_{i}-0, \Phi\left(\tau_{i}-0\right)\right),\right. \\
& u\left(\tau_{i}-0, x\left(\tau_{i}-0\right)+\kappa\right. \\
& \left.\left.\left(\left(\tau_{i}-0, \Phi\left(\tau_{i}-0\right)\right)\right)\right)\right) .
\end{aligned}
$$

We have the following estimation:

$$
\begin{aligned}
& \left|\mathscr{L} \kappa(s, x, y)-\mathscr{L} \kappa^{\prime}(s, x, y)\right| \\
& \leq \varepsilon(k+1) \\
& \times \int_{s}^{+\infty}|X(s, \tau)| \\
& \times\left|\kappa(\tau, \Phi(\tau))-\kappa^{\prime}(\tau, \Phi(\tau))\right| d \tau \\
& +\varepsilon(k+1) \sum_{s<\tau_{i}}\left|X\left(s, \tau_{i}\right)\right| \\
& \times \mid \kappa\left(\tau_{i}-0, \Phi\left(\tau_{i}-0\right)\right) \\
& -\kappa^{\prime}\left(\tau_{i}-0, \Phi\left(\tau_{i}-0\right)\right) \\
& \leq \varepsilon(k+1)\left\|\kappa-\kappa^{\prime}\right\| \\
& \times \int_{s}^{+\infty}|X(s, \tau)||y(\tau)-u(\tau, x(\tau))| d \tau \\
& +\varepsilon(k+1)\left\|\kappa-\kappa^{\prime}\right\| \sum_{s<\tau_{i}}\left|X\left(s, \tau_{i}\right)\right| \\
& \times\left|y\left(\tau_{i}-0\right)-u\left(\tau_{i}-0, x\left(\tau_{i}-0\right)\right)\right| \\
& \leq \varepsilon(k+1)\left\|\kappa-\kappa^{\prime}\right\| \frac{\nu|y-u(s, x)|}{1-\varepsilon \nu(k+1)} \\
& =k\left\|\kappa-\kappa^{\prime}\right\||y-u(s, x)| .
\end{aligned}
$$

Besides

$$
\begin{aligned}
\mid \mathscr{L} \kappa & (s, x, y) \mid+\varepsilon((k+1)\|\kappa\|+1) \\
& \times \int_{s}^{+\infty}|X(s, \tau)||y(\tau)-u(\tau, x(\tau))| d \tau \\
& +\varepsilon((k+1)\|\kappa\|+1) \\
& \times \sum_{s<\tau_{i}}\left|X\left(s, \tau_{i}\right)\right|\left|y\left(\tau_{i}-0\right)-u\left(\tau_{i}-0, x\left(\tau_{i}-0\right)\right)\right| \\
\leq & \frac{\varepsilon \nu((k+1)\|\kappa\|+1)}{1-\varepsilon v(k+1)}|y-u(s, x)| \\
= & \left(k\|\kappa\|+\frac{k}{k+1}\right)|y-u(s, x)| .
\end{aligned}
$$

If

$$
\|\kappa\| \leq k_{1}=\frac{k}{1-k^{2}}=\frac{\varepsilon \nu}{\sqrt{1-4 \varepsilon v}}
$$

then $\|\mathscr{L} \kappa\| \leq k_{1}$. We have that $\mathscr{L}$ is a contraction and $\mathscr{L} \kappa \epsilon$ $\mathfrak{M}_{1}$. It follows that there is only one solution satisfying the functional equation $\mathscr{L} \kappa=\kappa$. In addition for $t \geq s$

$$
\begin{aligned}
& \kappa(t, \Phi(t)) \\
& =\int_{t}^{+\infty} X(t, \tau)(f(\tau, \Phi(\tau)) \\
& -f(\tau, x(\tau)+\kappa(\tau, \Phi(\tau)), \\
& u(\tau, x(\tau)+\kappa(\tau, \Phi(\tau))))) d \tau \\
& +\sum_{t<\tau_{i}} X\left(t, \tau_{i}\right) \\
& \times\left(p_{i}\left(\Phi\left(\tau_{i}-0\right)\right)\right. \\
& -p_{i}\left(x\left(\tau_{i}-0\right)+\kappa\left(\tau_{i}-0, \Phi\left(\tau_{i}-0\right)\right),\right. \\
& \left.\left.u\left(\tau_{i}-0, x\left(\tau_{i}-0\right)+\kappa\left(\tau_{i}-0, \Phi\left(\tau_{i}-0\right)\right)\right)\right)\right) \\
& =X(t, s) \kappa(s, x, y) \\
& -\int_{s}^{t} X(t, \tau) \\
& \times(f(\tau, \Phi(\tau)) \\
& -f(\tau, x(\tau)+\kappa(\tau, \Phi(\tau)), \\
& u(\tau, x(\tau)+\kappa(\tau, \Phi(\tau))))) d \tau \\
& -\sum_{s<\tau_{i} \leq t} X\left(t, \tau_{i}\right)\left(p_{i}\left(\Phi\left(\tau_{i}-0\right)\right)\right. \\
& -p_{i}\left(x\left(\tau_{i}-0\right)+\kappa\left(\tau_{i}-0, \Phi\left(\tau_{i}-0\right)\right),\right. \\
& u\left(\tau_{i}-0, x\left(\tau_{i}-0\right)\right. \\
& \left.\left.\left.+\kappa\left(\tau_{i}-0, \Phi\left(\tau_{i}-0\right)\right)\right)\right)\right)
\end{aligned}
$$




$$
\begin{aligned}
& =-x(t)+X(t, s)(x+\kappa(s, x, y)) \\
& +\int_{s}^{t} X(t, \tau) \\
& \quad \times f(\tau, x(\tau)+\kappa(\tau, \Phi(\tau)), \\
& \quad u(\tau, x(\tau)+\kappa(\tau, \Phi(\tau)))) d \tau \\
& +\sum_{s<\tau_{i} \leq t} X\left(t, \tau_{i}\right) p_{i} \\
& \quad \times\left(x\left(\tau_{i}-0\right)+\kappa\left(\tau_{i}-0, \Phi\left(\tau_{i}-0\right)\right),\right. \\
& \left.\quad u\left(\tau_{i}-0, x\left(\tau_{i}-0\right)+\kappa\left(\tau_{i}-0, \Phi\left(\tau_{i}-0\right)\right)\right)\right) .
\end{aligned}
$$

Let

$$
\zeta(t)=x(t)+\kappa(t, \Phi(t))
$$

where $\zeta(s)=x+\kappa(s, x, y)$. It follows that $\zeta(t)$ is a solution of (12) and

$$
\begin{aligned}
& |\zeta(t)-x(t)| \\
& \quad=|\kappa(t, \Phi(t))| \leq \frac{\varepsilon v}{\sqrt{1-4 \varepsilon v}}|y(t)-u(t, x(t))| .
\end{aligned}
$$

This completes the proof of the theorem.

\section{Stability of the Impulsive Equations}

We assume in addition that

$$
\mu=\sup _{s} \int_{s}^{+\infty}|Y(t, s)| d t+\sup _{s} \sum_{\tau_{i}>s}\left|Y\left(\tau_{i}-0, s\right)\right|<+\infty .
$$

Note that in case $B(t)=B$ and $D_{i}=0$ we have $\mu=$ $\int_{0}^{+\infty}\left|e^{B t}\right| d t$

Theorem 6. Let $4 \varepsilon v<1$ and $2 \varepsilon \mu<1+\sqrt{1-4 \varepsilon v}$. Then the following estimation is valid:

$$
\begin{aligned}
\int_{s}^{+\infty} & |y(t)-u(t, x(t))| d t \\
& +\sum_{s<\tau_{i}}\left|y\left(\tau_{i}-0\right)-u\left(\tau_{i}-0, x\left(\tau_{i}-0\right)\right)\right| \\
& \leq \frac{\mu|y-u(s, x)|}{1-\varepsilon \mu(k+1)} .
\end{aligned}
$$

Proof. Since

$$
\begin{gathered}
k+1=\frac{1-\sqrt{1-4 \varepsilon v}}{2 \varepsilon \nu}=\frac{2}{1+\sqrt{1-4 \varepsilon v}}, \\
2 \varepsilon \mu<1+\sqrt{1-4 \varepsilon v}
\end{gathered}
$$

we get

$$
1-\varepsilon \mu(k+1)=1-\frac{2 \varepsilon \mu}{1+\sqrt{1-4 \varepsilon \mu}}>0
$$

From Theorem 4 of behaviour of solutions, we get inequality (40). Then doing the integration and summing up, inequality (55) is obtained.

Definition 7. A trivial solution of impulsive equation (1) is integral stable if for all $\varepsilon_{1}>0$ there exists a $\delta>0$ such that for all $|x|<\delta$ and $|y|<\delta$ and $t \geq s$ one has

$$
\int_{t}^{t+1}|x(\tau)| d \tau<\varepsilon_{1}, \quad \int_{t}^{t+1}|y(\tau)| d \tau<\varepsilon_{1} .
$$

Definition 8. A trivial solution of impulsive equation (1) is asymptotically integral stable if it is integral stable and if there exists a $\delta>0$ such that for all $|x|<\delta$ and $|y|<\delta$ one has

$$
\lim _{t \rightarrow+\infty} \int_{t}^{t+1}|x(\tau)| d \tau=0, \quad \lim _{t \rightarrow+\infty} \int_{t}^{t+1}|y(\tau)| d \tau=0 .
$$

Theorem 9. Let $4 v \varepsilon<1$ and $2 \varepsilon \mu<1+\sqrt{1-4 \varepsilon v}$. The trivial solution of impulsive equation (1) is integral stable, asymptotically integral stable, or integral unstable if and only if the trivial solution of impulsive equation (12) is integral stable, asymptotically integral stable, or integral unstable.

Proof. Suppose that the trivial solution of the system (12) is integral stable. Then for every $\varepsilon_{1}>0$, there is a $\delta_{1}>0$ such that for all $|\zeta(s)|<\delta_{1}$ and $t \geq s$ we have

$$
\int_{t}^{t+1}|\zeta(\tau)| d \tau<\frac{\varepsilon_{1}}{2}
$$

Let $|x|<\delta$ and $|y|<\delta$ where

$$
\delta<\min \left\{\frac{1-\varepsilon \mu(k+1)}{2 \mu\left(k_{1}+1\right)} \varepsilon_{1}, \delta_{1}\right\} .
$$

Then for $t \geq s$ we get

$$
\begin{aligned}
& \int_{t}^{t+1}|\zeta(\tau)-x(\tau)| d \tau \\
& \quad \leq k_{1} \int_{t}^{t+1}|y(\tau)-u(\tau, x(\tau))| d \tau \\
& \quad \leq \frac{\mu k_{1}(k+1)}{1-\varepsilon \mu(k+1)} \delta<\frac{\varepsilon_{1}}{2}, \\
& \int_{t}^{t+1} \quad|y(\tau)-u(\tau, \zeta(\tau))| d \tau \\
& \quad \leq \int_{t}^{t+1}|y(\tau)-u(\tau, x(\tau))| d \tau \\
& \quad+\int_{t}^{t+1}|u(\tau, x(\tau))-u(\tau, \zeta(\tau))| d \tau \\
& \leq\left(1+k k_{1}\right) \int_{t}^{t+1}|y(\tau)-u(\tau, x(\tau))| d \tau \\
& \leq \frac{\mu\left(1+k k_{1}\right)(k+1)}{1-\varepsilon \mu(k+1)} \delta<\frac{\varepsilon_{1}}{2} .
\end{aligned}
$$


Therefore

$$
\begin{aligned}
& \int_{t}^{t+1}|x(\tau)| d \tau \\
& \quad \leq \int_{t}^{t+1}|x(\tau)-\zeta(\tau)| d \tau \\
& \quad+\int_{t}^{t+1}|\zeta(\tau)| d \tau<\varepsilon_{1}, \\
& \int_{t}^{t+1} \quad|y(\tau)| d \tau \\
& \leq \int_{t}^{t+1}|y(\tau)-u(\tau, \zeta(\tau))| d \tau \\
& \quad+\int_{t}^{t+1}|u(\tau, \zeta(\tau))| \\
& <\frac{(k+1) \varepsilon_{1}<\varepsilon_{1} .}{2}
\end{aligned}
$$

Suppose that the trivial solution of the system (12) is asymptotically integral stable. Then

$$
\lim _{t \rightarrow+\infty} \int_{t}^{t+1}|\zeta(\tau)| d \tau=0
$$

It follows that

$$
\begin{aligned}
& \lim _{t \rightarrow+\infty} \int_{t}^{t+1}|x(\tau)| d \tau \\
& \leq \lim _{t \rightarrow+\infty} \int_{t}^{t+1}|x(\tau)-\zeta(\tau)| d \tau \\
& \quad+\lim _{t \rightarrow+\infty} \int_{t}^{t+1}|\zeta(\tau)| d \tau=0, \\
& \lim _{t \rightarrow+\infty} \int_{t}^{t+1}|y(\tau)| d \tau \\
& \leq \lim _{t \rightarrow+\infty} \int_{t}^{t+1}|y(\tau)-u(\tau, x(\tau))| d \tau \\
& \quad+\lim _{t \rightarrow+\infty} \int_{t}^{t+1}|u(\tau, x(\tau))| d \tau=0,
\end{aligned}
$$

taking into account that

$$
|u(t, x(t))| \leq k|x(t)|
$$

If the trivial solution of (12) is integral unstable, then the trivial solution of (1) is integral unstable.

If the trivial solution of (1) is integral stable or asymptotically integral stable, then the trivial solution of (12) is also integral stable or asymptotically integral stable.

Let the trivial solution of (1) be integral unstable; then the trivial solution of (12) is integral unstable. Otherwise as before it follows that the trivial solution of (1) is integral stable. We get a contraction. The theorem is proven.
Theorem 10. Assume that the estimates

$$
\begin{aligned}
& \mu(\beta)=\sup _{s} \int_{s}^{+\infty}|Y(t, s)| e^{\beta(t-s)} d t \\
& \quad+\sup _{s} \sum_{\tau_{i}>s}\left|Y\left(\tau_{i}-0, s\right)\right| e^{\beta\left(\tau_{i}-s\right)}<+\infty, \\
& |Y(t, s)| e^{\beta(t-s)} \leq M(\beta) \quad \forall t \geq s
\end{aligned}
$$

are satisfied for some $\beta \geq 0$. If $4 \varepsilon \nu<1$ and $2 \varepsilon \mu(\beta)<1+$ $\sqrt{1-4 \varepsilon v}$, then

$$
\begin{aligned}
& \quad|y(t)-u(t, x(t))| \\
& \quad \leq \alpha(\beta) e^{-\beta(t-s)}|y-u(s, x)| \quad \text { for } t \geq s .
\end{aligned}
$$

Proof. From Theorem 4 of behaviour of solutions, we get inequality (40). Multiplying by $e^{\beta(t-s)}$ and doing the integration and summing up, the inequality

$$
\begin{aligned}
\int_{s}^{+\infty} & e^{\beta(t-s)}|y(t)-u(t, x(t))| d t \\
& +\sum_{s<\tau_{i}} e^{\beta\left(\tau_{i}-s\right)}\left|y\left(\tau_{i}-0\right)-u\left(\tau_{i}-0, x\left(\tau_{i}-0\right)\right)\right| \\
& \leq \frac{\mu(\beta)|y-u(s, x)|}{1-\varepsilon \mu(\beta)(k+1)}
\end{aligned}
$$

is obtained.

Then from inequality (40) for $t \geq s$ we get the estimation

$$
\begin{aligned}
& |y(t)-u(t, x(t))| \\
& \leq M(\beta) e^{-\beta(t-s)}|y-u(s, x)| \\
& +\varepsilon(k+1) M(\beta) e^{-\beta(t-s)} \\
& \quad \times\left(\int_{s}^{+\infty} e^{\beta(r-s)}|y(r)-u(r, x(r))| d r\right. \\
& \left.\quad+\sum_{s<\tau_{i}}\left|y\left(\tau_{i}-0\right)-u\left(\tau_{i}-0, x\left(\tau_{i}-0\right)\right)\right|\right) \\
& \leq M(\beta) e^{-\beta(t-s)}\left(1+\frac{\varepsilon \mu(\beta)(k+1)}{1-\varepsilon \mu(\beta)(k+1)}\right)|y-u(s, x)| \\
& =\alpha(\beta) e^{-\beta(t-s)}|y-u(s, x)| .
\end{aligned}
$$

Theorem 11. Let $4 v \varepsilon<1,2 \varepsilon \mu<1+\sqrt{1-4 \varepsilon v}$,

$$
\begin{gathered}
|y(t)-u(t, x(t))| \leq \alpha|y-u(s, x)| \quad \text { if } t \geq s, \\
\lim _{t \rightarrow+\infty}(y(t)-u(t, x(t)))=0 .
\end{gathered}
$$

The trivial solution of impulsive equation (1) is stable, asymptotically stable, or unstable if and only if the trivial solution of impulsive equation (12) is stable, asymptotically stable, or unstable. 
Proof. Suppose that the trivial solution of the system (12) is stable. Then for every $\varepsilon_{1}>0$, there is a $\delta_{1}>0$ such that for all $|\zeta(s)|<\delta_{1}$ and $t \geq s$ we have $|\zeta(t)|<\varepsilon_{1} / 2$.

Let $|x|<\delta$ and $|y|<\delta$ where

$$
\delta<\min \left\{\frac{\varepsilon_{1}}{2 \alpha\left(k_{1}+1\right)(k+1)}, \delta_{1}\right\} .
$$

Then for $t \geq s$ we get

$$
\begin{aligned}
&|\zeta(t)-x(t)| \leq k_{1}|y(t)-u(t, x(t))| \\
& \leq \alpha k_{1}(k+1) \delta<\frac{\varepsilon_{1}}{2}, \\
&|y(t)-u(t, \zeta(t))| \\
& \leq|y(t)-u(t, x(t))| \\
& \quad+|u(t, x(t))-u(t, \zeta(t))| \\
& \leq\left(1+k k_{1}\right)|y(t)-u(t, x(t))| \\
& \leq \alpha\left(1+k k_{1}\right)(k+1) \delta<\frac{\varepsilon_{1}}{2} .
\end{aligned}
$$

Therefore

$$
\begin{aligned}
|x(t)| & \leq|x(t)-\zeta(t)|+|\zeta(t)|<\varepsilon_{1}, \\
|y(t)| & \leq|y(t)-u(t, \zeta(t))|+|u(t, \zeta(t))| \\
& <\frac{(k+1) \varepsilon_{1}}{2}<\varepsilon_{1} .
\end{aligned}
$$

Suppose that the trivial solution of the system (12) is asymptotically stable. Then

$$
\lim _{t \rightarrow+\infty} \zeta(t)=0
$$

It follows that

$$
\begin{gathered}
\lim _{t \rightarrow+\infty} x(t)=\lim _{t \rightarrow+\infty}(x(t)-\zeta(t))+\lim _{t \rightarrow+\infty} \zeta(t)=0, \\
\lim _{t \rightarrow+\infty} y(t)=\lim _{t \rightarrow+\infty}(y(t)-u(t, x(t))) \\
+\lim _{t \rightarrow+\infty} u(t, x(t))=0 .
\end{gathered}
$$

If the trivial solution of (12) is unstable, then the trivial solution of (1) is unstable.

If the trivial solution of (1) is stable or asymptotically stable, then the trivial solution of (12) is also stable or asymptotically stable.

Let the trivial solution of (1) be unstable; then the trivial solution of (12) is unstable. Otherwise as before it follows that the trivial solution of (1) is stable. We get a contraction. The theorem is proven.

Remark 12. Let $\eta(t)=|y(t)-u(t, x(t))|$ be uniformly continuous on $t \in[s,+\infty)$ and let improper integral $\int_{s}^{+\infty} \eta(t) d t$ converge. Then $\lim _{t \rightarrow+\infty} \eta(t)=0$ [13, page 32].
Remark 13. If we replace assumption (54) by the stronger one

$$
\begin{aligned}
\mu_{1}= & \int_{0}^{+\infty} \sup _{s}|Y(t+s, s)| d t \\
& +\sum_{\tau_{i}>0} \sup _{s}\left|Y\left(s+\tau_{i}-0, s\right)\right|<+\infty,
\end{aligned}
$$

then for $t \geq s$ it is possible to prove that $|Y(t, s)| \leq$ $K \exp (-\lambda(t-s))$, where $K \geq 1$ and $\lambda>0$. Further, if $\varepsilon>0$ is sufficiently small, then using Gronwall's lemma for all $t \geq s$ the following estimation is valid:

$$
|y(t)-u(t, x(t))| \leq K e^{-\lambda_{1}(t-s)}|y-u(s, x)|,
$$

where $0<\lambda_{1}<\lambda$.

\section{Acknowledgment}

This work was partially supported by the Grant no. 345/2012 of the Latvian Council of Science.

\section{References}

[1] V. A. Pliss, "A reduction principle in the theory of stability of motion,” Izvestiya Akademii Nauk SSSR. Seriya Matematicheskaya, vol. 28, no. 6, pp. 1297-1324, 1964 (Russian).

[2] B. Aulbach, "A reduction principle for nonautonomous differential equations," Archiv der Mathematik, vol. 39, no. 3, pp. 217232, 1982.

[3] C. Pötzsche, Geometric Theory of Discrete Nonautonomous Dynamical Systems, Springer, Heidelberg, Germany, 2010.

[4] O. B. Lykova, "The reduction principle in a Banach space," Ukrainian Mathematical Journal, vol. 23, pp. 391-397, 1971 (Russian).

[5] A. Reinfelds and K. Janglajew, "Reduction principle in the theory of stability of difference equations," Discrete and Continuous Dynamical Systems, supplement, pp. 864-874, 2007.

[6] D. D. Bainov, S. I. Kostadinov, and N. Van Minh, Dichotomies and Integral Manifolds of Impulsive Differential Equations, Science Culture Technology Publishing, Singapore, 1994.

[7] A. Reinfelds, "A reduction theorem for systems of differential equations with impulse effect in a Banach space," Journal of Mathematical Analysis and Applications, vol. 203, no. 1, pp. 187210, 1996.

[8] W. A. Coppel, Dichotomies in Stability Theory, Springer, Berlin, Germany, 1978.

[9] P. E. Kloeden and M. Rasmussen, Nonautonomous Dynamical Systems, American Mathematical Society, Providence, RI, USA, 2011.

[10] L. Ya. Adrianova, Introduction to Linear Systems of Differential Equations, American Mathematical Society, Providence, RI, USA, 1995.

[11] V. Lakshmikantham, D. D. Baĭnov, and P. S. Simeonov, Theory of Impulsive Differential Equations, World Scientific, Singapore, 1989.

[12] A. M. Samoilenko and N. A. Perestyuk, Impulsive Differential Equations, World Scientific, Singapore, 1985.

[13] W. J. Kaczor and M. T. Nowak, Problems in Mathematical Analysis III. Integration, American Mathematical Society, Providence, RI, USA, 2003. 


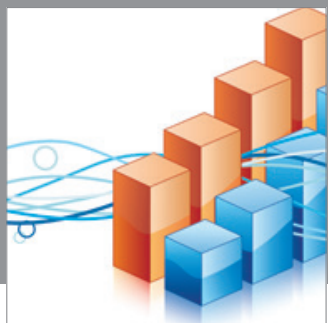

Advances in

Operations Research

mansans

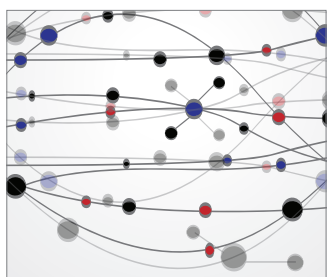

The Scientific World Journal
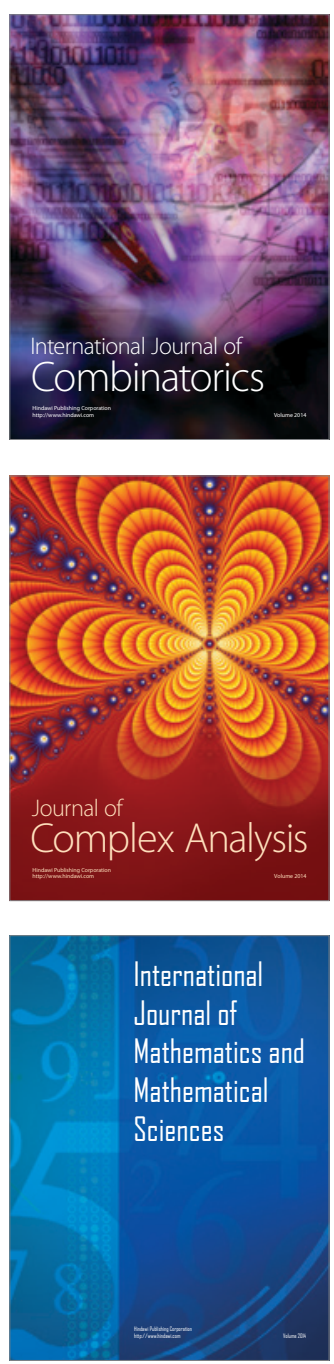
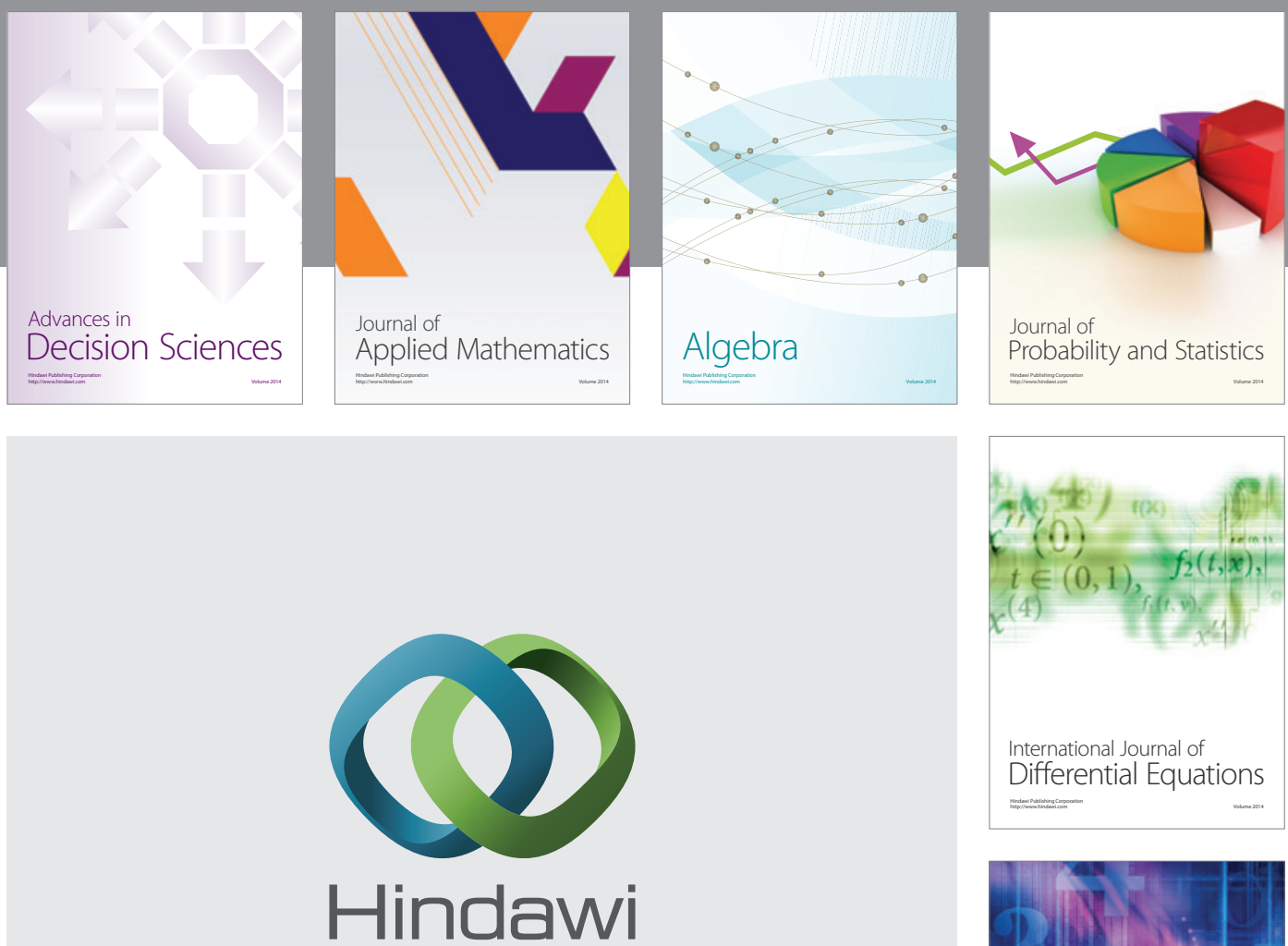

Submit your manuscripts at http://www.hindawi.com
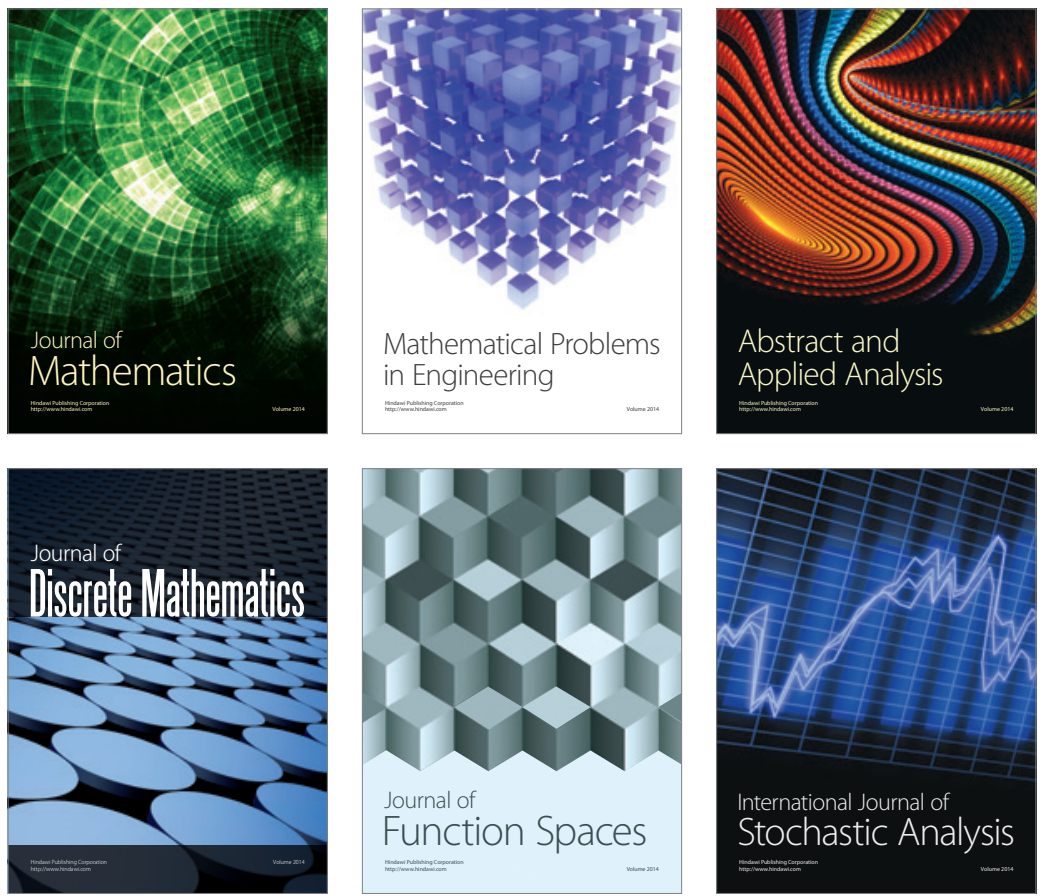

Journal of

Function Spaces

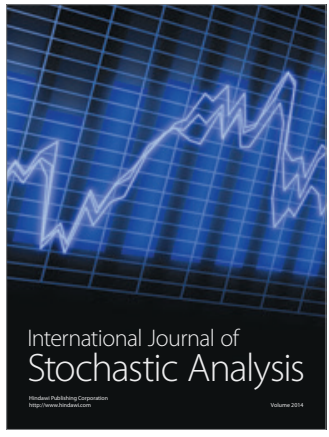

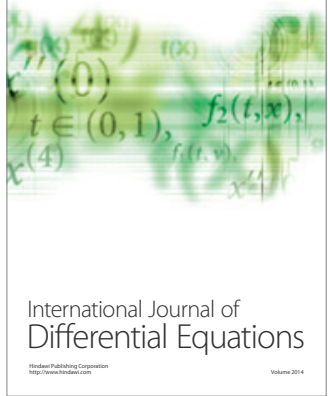
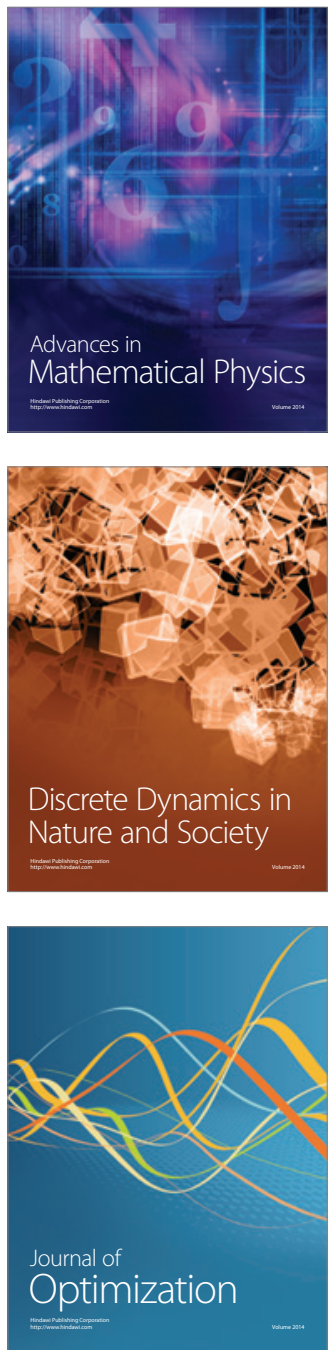